\title{
Subdiffraction optical manipulation of the charge state of nitrogen vacancy center in diamond
}

\author{
Xiangdong Chen ${ }^{1,2, *}$, Changling Zou ${ }^{1,2, *}$, Zhaojun Gong ${ }^{1,2}$, Chunhua Dong ${ }^{1,2}$, Guangcan Guo ${ }^{1,2}$ \\ and Fangwen Sun ${ }^{1,2}$
}

As a potential candidate for quantum computation and metrology, the nitrogen vacancy (NV) center in diamond presents both challenges and opportunities resulting from charge-state conversion. By utilizing different lasers for the photon-induced charge-state conversion, we achieved subdiffraction charge-state manipulation. The charge-state depletion (CSD) microscopy resolution was improved to $4.1 \mathrm{~nm}$ by optimizing the laser pulse sequences. Subsequently, the electron spin-state dynamics of adjacent NV centers were selectively detected via the CSD. The experimental results demonstrated that the CSD can improve the spatial resolution of the measurement of NV centers for nanoscale sensing and quantum information.

Light: Science \& Applications (2015) 4, e230; doi:10.1038/Isa.2015.3; published online 2 January 2015

Keywords: charge state; NV center; photon ionization; super-resolution microscopy

\section{INTRODUCTION}

Because of the stable fluorescence and long coherence time of its spin state, the negatively charged nitrogen vacancy $\left(\mathrm{NV}^{-}\right)$center in diamond has been studied extensively over the past decade. This defect has the potential to be used for quantum computation, ${ }^{1-4}$ nanoscale metrology ${ }^{5-8}$ and biological imaging. ${ }^{9-11}$ To further extend the study of the interaction between a multi-NV center and the nanoscale sensing with the NV center, it is necessary to detect and control the NV center spin-state dynamics with high spatial resolution. ${ }^{7,12-14}$ Therefore, many optical super-resolution microscopy techniques have been developed to detect single NV centers. ${ }^{13-17}$ Among these methods, stimulated emission depletion (STED) microscopy ${ }^{12,18-20}$ is one of the most promising. This method utilizes a doughnut-shaped laser to produce the position-dependent stimulated emission, which changes the fluorescence signal. With STED, the electron spin resonance signals of $\mathrm{NV}$ centers have been detected with a resolution lower than the diffraction limit. ${ }^{12,21,22}$

A new super-resolution microscopy technique was recently developed by Han et al. ${ }^{15}$ The authors replaced the stimulated excitation of STED with the dark-state pumping of NV centers. The dark state was later proven to be the neutral charge $\mathrm{NV}$ center $\left(\mathrm{NV}^{0}\right)$ by other groups. ${ }^{23-25}$ The charge-state conversion results in a change of the local field in diamond ${ }^{26}$ and the spectral diffusion of the $\mathrm{NV}$ center. $^{24,27}$ For high-fidelity quantum manipulation, the charge state should be well controlled. ${ }^{28}$ Based on the mechanism of charge-state conversion and super-resolution microscopy, ${ }^{25,29,30}$ we demonstrated the optical manipulation of the charge state of an NV center with subdiffraction resolution. By changing the duration and power of laser pulses, we optimized the charge-state depletion (CSD) microscopy to improve the resolution to $4.1 \mathrm{~nm}$ without oil immersion lens. With the charge-state manipulation, we were able to detect the electron spin-state dynamics of NV centers with subdiffraction resolution, which can be used for nanoscale sensing of electromagnetic fields and biological molecules. Because charge-state conversion changes the local field in diamond, ${ }^{2,26}$ the CSD microscopy method can potentially be used for the study of spin-state quantum coherent dynamics and the interaction between coupled spin systems in diamond. ${ }^{2,13,31}$

\section{MATERIALS AND METHODS}

The NV center in diamond consists of a substitutional nitrogen atom and an adjacent vacancy. Two charge states are usually observed: $\mathrm{NV}^{0}$ and $\mathrm{NV}^{-}$(Figure 1a). Both $\mathrm{NV}^{-}$and $\mathrm{NV}^{0}$ are stable in the absence of optical excitation. ${ }^{15,32}$ The electron spin of the $\mathrm{NV}^{-}$ground state can be optically initialized and detected. ${ }^{1}$ The coherence time of the spin state could exceed several milliseconds. ${ }^{33}$ However, the $\mathrm{NV}^{0}$ charge state does not exhibit such properties. As observed in Figure 1c, the photons of $\mathrm{NV}^{0}$ and $\mathrm{NV}^{-}$are different in wavelength. Therefore, we are able to distinguish the two charge states by measuring the NV center fluorescence. The conversion between these two charge states can be observed as the fluorescence on and off processes, which can be used for reversible saturable optical fluorescence transition microscopy.

The samples used in this experiment were $\langle 100\rangle$ surface diamond plates with nitrogen concentrations lower than $5 \mathrm{ppb}$. The NV centers were produced by $\mathrm{N}^{+}$ion implantation and subsequent annealing. The optical system used to locate and detect the NV center is shown in Figure 1b. Laser pulses with various wavelengths and beam shapes

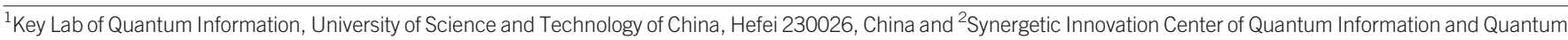
Physics, University of Science and Technology of China, Hefei 230026, China

*These authors contributed equally

Correspondence: FW Sun, Key Lab of Quantum Information, University of Science and Technology of China, Hefei 230026, China

E-mail: fwsun@ustc.edu.cn
}

Received 11 September 2014; revised 29 October 2014; accepted 30 October 2014; accepted article preview online 10 November 2014 
575-nm ZPL
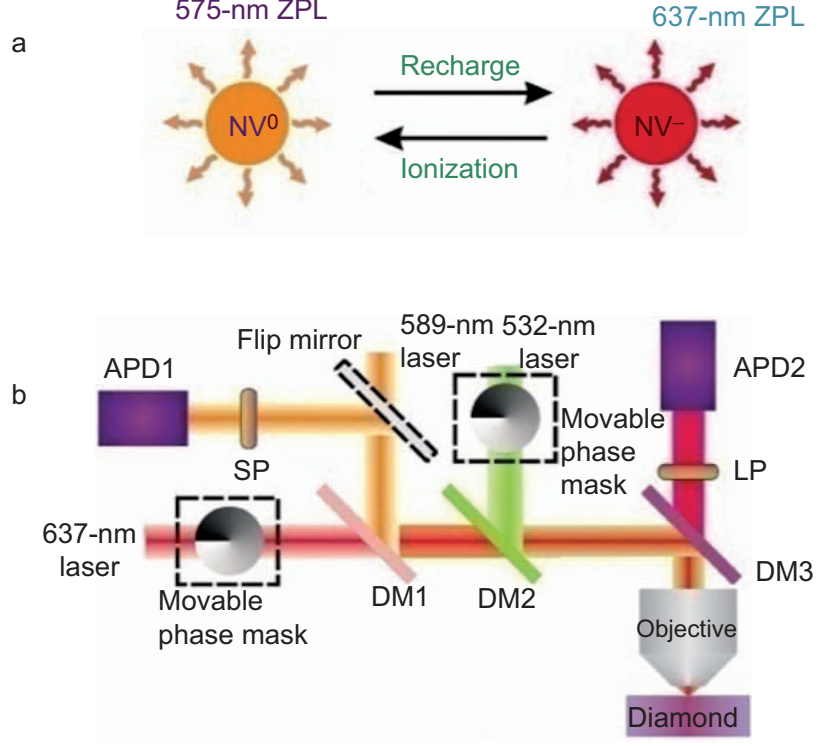

C

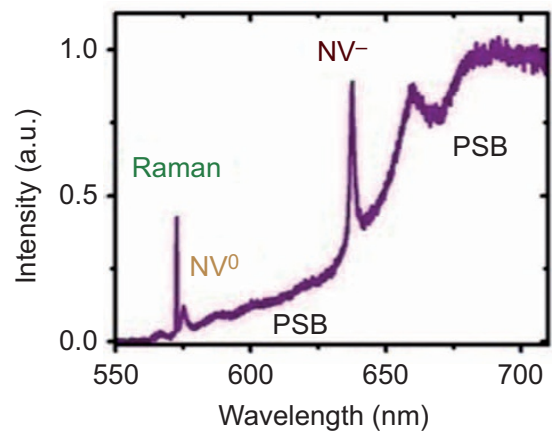

e

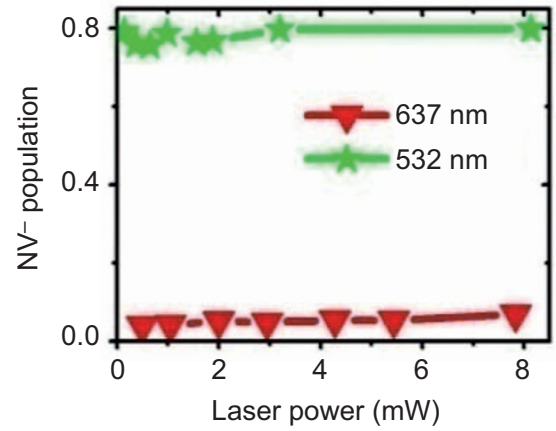

d

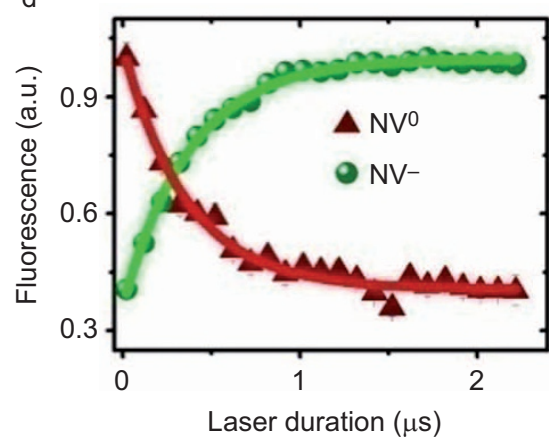

f

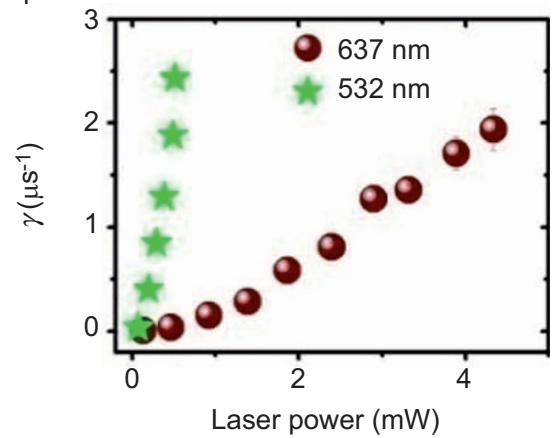

Figure 1 (a) A sketch depicting the charge-state conversion between $\mathrm{NV}^{-}$and $\mathrm{NV}^{0}$. (b) Schematics of the experimental configuration. The lasers and fluorescence emission were combined and split using DM1 (edge wavelength $605 \mathrm{~nm}$ ), DM2 (edge wavelength $536.8 \mathrm{~nm}$ ) and DM3 (edge wavelength $658.8 \mathrm{~nm}$ ). The fluorescence of $\mathrm{NV}^{-}$was detected by APD2 with a long-pass filter (LP, edge wavelength $668.9 \mathrm{~nm}$ ), and the fluorescence of NV was detected by APD 1 with a short-pass filter (SP, edge wavelength $599 \mathrm{~nm}$ ). Two phase masks were used to produce the doughnut-shaped laser beams. The 637-nm and 532-nm lasers were used to initialize or switch the charge state, and the 589-nm laser is for the detection of the charge state. (c) Fluorescence spectrum of the NV center ensemble excited by a 532-nm laser at temperature approximately $250 \mathrm{~K}$. The charge states of NV are marked by different ZPLs. The PSB of each charge state presents photons at longer wavelength. (d) Charge-state conversion process of NV ensemble pumped by a 532-nm laser. The charge states were initialized using a 637-nm laser. (e and f) Power dependence of the steady charge state population and charge-state conversion rates $\left(\gamma=\gamma_{\mathrm{r}}+\gamma_{\mathrm{i}}\right)$. All of the lasers were Gaussian beam lasers. The charge-state conversion rates were obtained by fitting the results of $\mathrm{NV}^{-}$fluorescence in $\mathbf{d}$ with Equation (3). APD, avalanche photodiode; DM, long-pass dichroic mirrors; NV, nitrogen vacancy; PSB, phonon sideband; ZPL, zero phonon line.

were switched using acoustic optical modulators and then focused on the sample through an objective (numerical aperture $=0.9$ ). The fluorescence emission of $\mathrm{NV}^{0}$ and $\mathrm{NV}^{-}$was split using a long-pass dichroic mirror and was further filtered using a short-pass filter and a long-pass filter, respectively.

\section{RESULTS AND DISCUSSION}

Optical manipulation of the charge state

Various methods have been developed to control the charge states of NV centers, ${ }^{25,27,30,34-37}$ with the most convenient method being high-speed optical manipulation. To demonstrate photon-induced 
charge-state conversion between $\mathrm{NV}^{0}$ and $\mathrm{NV}^{-}$, the $\mathrm{NV}$ center ensemble fluorescence emission of both $\mathrm{NV}^{0}$ and $\mathrm{NV}^{-}$charge states was detected. As demonstrated in Figure 1d, the fluorescence intensities of the two charge states changed in opposite manners when the $\mathrm{NV}$ center was pumped with a $0.68-\mathrm{mW}, 532-\mathrm{nm}$ laser. The fluorescence changing rates of the two charge states were almost the same (approximately $2.7 \mu \mathrm{s}^{-1}$ in Figure 1d). In contrast, the excited state lifetimes of $\mathrm{NV}^{-}$and $\mathrm{NV}^{0}$ were both approximately $13 \mathrm{~ns}$ in our experiments. As the fluorescence intensity is directly proportion to the population of charge state, the fluorescence changing rate can be simply presented as the charge-state conversion rate. This result indicates that the charge state can be switched from $\mathrm{NV}^{0}$ to $\mathrm{NV}^{-}$using a 532-nm laser.

Both the ionization $\left(\mathrm{NV}^{-}\right.$to $\left.\mathrm{NV}^{0}\right)$ and recharging $\left(\mathrm{NV}^{0}\right.$ to $\left.\mathrm{NV}^{-}\right)$ processes can be excited using the same laser. The steady-state population is determined by both the ionization and recharging rates, which are significantly changed by the wavelength and power of the laser. We measured the charge-state population using the single-shot charge-state readout method. ${ }^{25,30}$ In our experiment, the highest population of $\mathrm{NV}^{-}$(approximately 75\%) was obtained by excitation with a 532-nm laser, while the lowest population of $\mathrm{NV}^{-}$(approximately 5\%) was obtained using a 637-nm laser (Figure 1e). Therefore, these two lasers were selected to initialize and change the charge-state population of the NV centers. The fidelity of charge-state initialization would be higher than that in Han's work, ${ }^{15}$ where a 473/592-nm laser was used to initialize the NV center charge state. Previous experiments demonstrated that the charge-state conversion is mainly a two-photon process with a laser wavelength longer than $500-\mathrm{nm} .^{25,30,36}$ The NV is first pumped to the excited state of $\mathrm{NV}^{-}$or $\mathrm{NV}^{0}$; then, an electron is released to the conduction band during ionization or captured from the valance band during recharging. ${ }^{24}$ The nonlinear power dependence of the conversion rates in Figure if was obtained by repeating the measurement of Figure 1d with different laser powers. The results demonstrate that the charge-state conversions with 532 and $637 \mathrm{~nm}$ are two-photon processes (see Supplementary Information for details). ${ }^{23,25,30}$

Simply, we can write the rate equations during charge-state conversion as

$$
\begin{aligned}
& \dot{\rho}_{0}=-\gamma_{\mathrm{r}} \rho_{0}+\gamma_{\mathrm{i}} \rho_{-} \\
& \dot{\rho}_{-}=+\gamma_{\mathrm{r}} \rho_{0}-\gamma_{\mathrm{i}} \rho_{-}
\end{aligned}
$$

where $\rho_{0}$ and $\rho_{-}$are the population of $\mathrm{NV}^{0}$ and $\mathrm{NV}^{-}$, respectively, and $\gamma_{r}$ and $\gamma_{i}$ are the recharging and ionization conversion rates, respectively. If only the photons of $\mathrm{NV}^{-}$are detected, the fluorescence intensity can be written as

$$
\eta \propto \rho_{-}=\rho_{-, \mathrm{st}}+\left(\rho_{-, \mathrm{in}}-\rho_{-, \mathrm{st}}\right) e^{-\gamma \tau}
$$

where $\gamma=\gamma_{\mathrm{r}}+\gamma_{\mathrm{i}}$ is the experimentally measured fluorescence changing rate, as observed in Figure 1d. $\rho_{-, s t}=\gamma_{r} /\left(\gamma_{r}+\gamma_{i}\right)$ is the steady-state $\mathrm{NV}^{-}$population with laser pumping, where $\rho_{-, \text {in }}$ is the initial $\mathrm{NV}^{-}$ population. And, $\tau$ is the laser duration. Therefore, the high-spatialresolution charge-state manipulation requires a position-dependent charge-state conversion rate $\gamma(\boldsymbol{r})$ or a position-dependent steady-state population $\rho_{-, s t}(r)$. According to the experimental results in Figure $1 \mathrm{e}$, the steady-state populations change little with the power of 532-nm and 637-nm lasers. In contrast, the charge-state conversion rates increase with the power of the laser (Figure 1f). Hence, a doughnut-shaped laser beam is used to produce the position-dependent charge-state conversion rate. The power intensity at the beam center approaches zero. We considered two NV centers, one at the beam center with position $\boldsymbol{r}_{0}$ and the other at $\boldsymbol{r}_{0}+\Delta \boldsymbol{r}$. Because $\gamma\left(\boldsymbol{r}_{0}\right)=0$, the fluorescence difference between the two NV centers is $\left|\eta_{\boldsymbol{r}_{0}}-\eta_{\boldsymbol{r}_{0}+\Delta \boldsymbol{r}}\right| \propto\left|\rho_{-, \text {in }}-\rho_{-, \text {st }}\right| \cdot\left(1-e^{-\gamma\left(\boldsymbol{r}_{0}+\Delta \boldsymbol{r}\right) \tau}\right)$. Therefore, for any $\Delta \boldsymbol{r}$, the fluorescence difference $\left|\eta_{\boldsymbol{r}_{0}}-\eta_{\boldsymbol{r}_{0}+\Delta \boldsymbol{r}}\right|$ is saturable and can be increased with the power and duration of the laser.

\section{CSD microscopy}

Next, we will outline the procedure for subdiffraction CSD microscopy imaging. Three different laser pulses were used in the experiment, as shown in Figure 2a and 2b. First, a Gaussian beam (G) laser was used to initialize the charge state in the focus region. Second, a doughnut-shaped (D) laser pulse was applied. The wavelength of the $D$ laser was different from that of the G laser. This laser was able to deplete the $\mathrm{NV}^{0}$ or $\mathrm{NV}^{-}$charge state except for the NV at the beam center. Finally, the charge state was detected with a $0.1-\mathrm{mW}, 589-\mathrm{nm}$ laser. The duration of the 589-nm laser was $5 \mu$ s. The microscopy image was obtained by detecting the fluorescence of $\mathrm{NV}^{-}$and scanning the sample with a piezo stage. All of the lasers were circularly polarized to obtain the same conversion rates for the NV centers with different symmetry axes.

For high-resolution ionization manipulation (change NV center to a high $\mathrm{NV}^{0}$ population state), we used a $637-\mathrm{nm}$ G laser and a $532-\mathrm{nm} \mathrm{D}$ laser (Figure 2a). The 637-nm laser initialized the NV with an $\mathrm{NV}^{0}$ population of approximately 95\%. The 532-nm D laser converted $\mathrm{NV}^{0}$ into $\mathrm{NV}^{-}$with a position-dependent conversion rate. A typical microscopy image is presented in Figure 2a. NV centers appear as dark spots in the image. In contrast, using a 637-nm D laser and a 532-nm G laser, we achieved higher solution recharging manipulation (change NV center to a high $\mathrm{NV}^{-}$population state) (Figure $2 \mathrm{~b}$ ). The NV center would be initialized to $\mathrm{NV}^{-}$with a probability of approximately $75 \%$ and converted into $\mathrm{NV}^{0}$ with a position-dependent rate. $\mathrm{NV}$ centers appear as bright points in the fluorescence microscope image. To distinguish the imaging using different laser sequences, the CSD microscopy using a 637-nm D laser can be termed as recharging-CSD (rCSD), and the 532-nm D laser CSD is termed as ionization-CSD (iCSD).

The effective point-spread-function of the CSD image is represented by $h(r)=h_{\text {det }}(r) \rho_{-}(r)$, where $h_{\text {det }}(r)$ is the detection pointspread-function. For the two-photon charge-state conversion, the charge-state conversion rate should be quadratically dependent on the power of the laser. We simply write the conversion rate as $\gamma \approx \alpha I^{2}$ (see details in Supplementary Information), where $I$ is the power of laser. The simplest function to depict the detection pointspread-function and the doughnut conversion laser intensity is the standing wavefunction: ${ }^{38,39}$

$$
\begin{aligned}
& h_{\text {det }}(r)=C \cos ^{2}\left(\pi r / \omega_{\text {det }}\right) \\
& I_{\mathrm{D}}(r)=I_{\max } \sin ^{2}\left(\pi r / \omega_{\mathrm{D}}\right)
\end{aligned}
$$

For simplicity, we assume $\omega_{\text {det }} \approx \omega_{\mathrm{D}}$ (the width of the detection Gaussian beam laser approximates the width of the D laser). The resolution of CSD can be approximated by a Taylor series:

$$
\Delta r=\frac{2 \omega_{\mathrm{D}}}{\pi} \sqrt{\frac{-3+\sqrt{3} \sqrt{6 \beta+1}}{2(3 \beta-1)}}
$$

where $\beta=\alpha I_{\max }^{2} \tau$, which indicates that increasing the power and duration of the $\mathrm{D}$ laser could improve the CSD resolution, as 
a
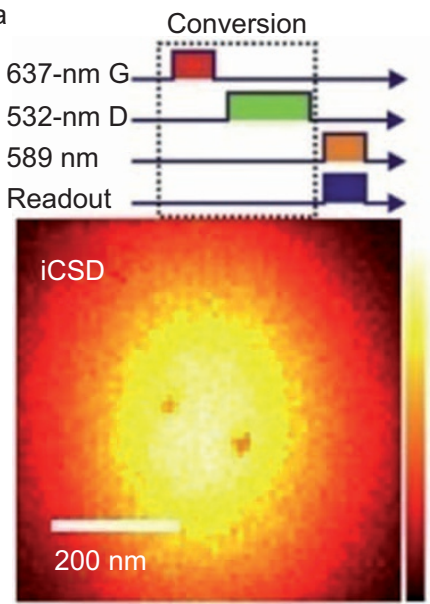

b

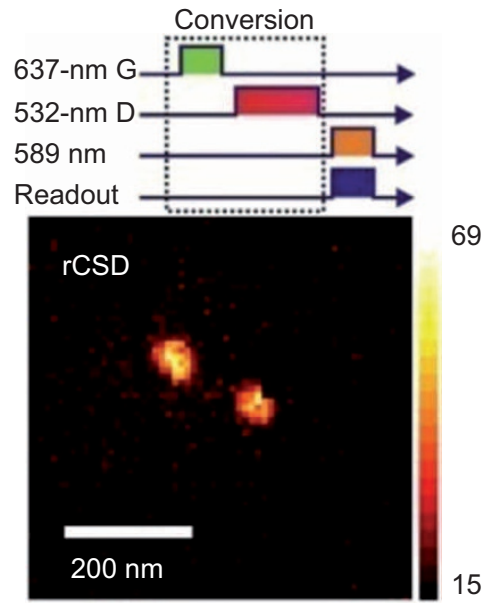

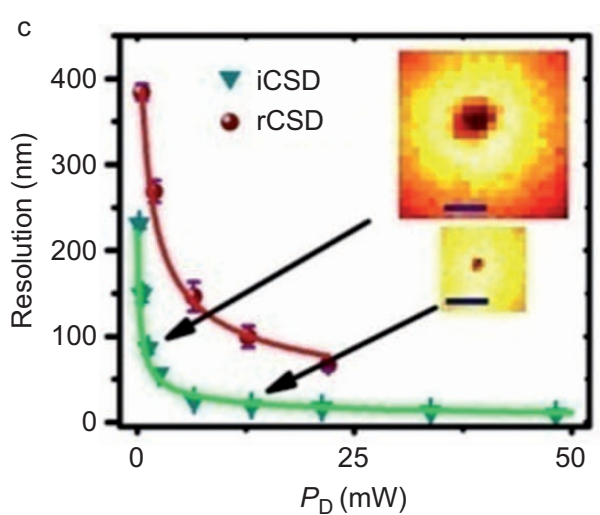

752

36
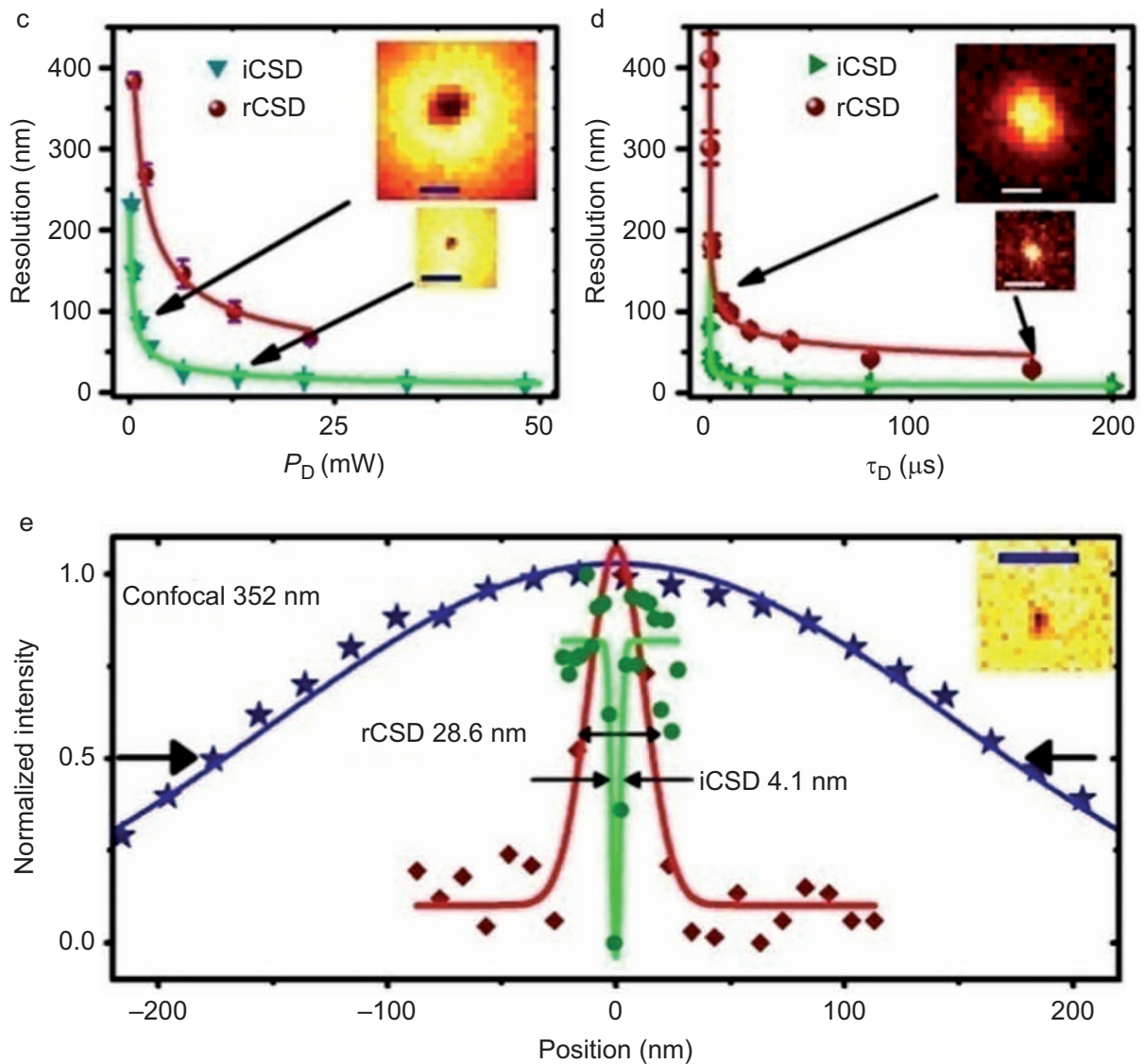

Figure 2 ( $\mathbf{a}$ and $\mathbf{b}$ ) Laser sequences and example images for the high-resolution charge-state ionization and recharging manipulation, respectively. (c and $\mathbf{d}$ ) The resolution (presented by FWHM) improved by increasing the D laser power or duration. The solid lines are the fits with Equation (6). The duration of the D lasers in $\mathbf{c}$ is $40 \mu \mathrm{s}$. The D laser in $\mathbf{d}$ is a 34-mW, 532-nm laser for iCSD and a22-mW, 637-nm laser for rCSD. The insets in $\mathbf{c}$ and $\mathbf{d}$ are the images of iCSD with different powers and rCSD with different durations, respectively. The scale bar is $100 \mathrm{~nm}$. (e) The profiles of confocal (blue star dots) and CSD images. In our experiment, the best rCSD resolution was approximately $28.6 \mathrm{~nm}$ (22-mW, 637-nm doughnut laser with 160- $\mu$ s duration), shown as red square dots. And the best iCSD resolution was $4.1 \mathrm{~nm}$ (48-mW, 532-nm doughnut laser with 200-us resolution), shown as the green circular dots. The inset shows the iCSD image of the best resolution. The scale bar is $30 \mathrm{~nm}$. FWHM, full width at half maximum; iCSD, ionization-charge-state depletion; rCSD, recharging-charge-state depletion.

observed in Figure 2c and 2d. In addition, the microscopy resolution is theoretically unlimited. For the same power, the charge-state conversion rate of the 532-nm laser is higher than that of the 637$\mathrm{nm}$ laser, as observed in Figure 1f, which might result from the wavelength-dependent absorption cross-section of the NV center at room temperature. ${ }^{25,36}$ Therefore, the resolution of iCSD is better than that of rCSD for the same power and duration of the D laser.

In reality, the improvement of the CSD resolution remains limited by the power of the laser and stability of the piezo stage. Figure $2 \mathrm{e}$ shows the best resolution in our experiment. The inset presents the 
iCSD image with the FWHMs (full widths at half maximum) in two directions, approximately $4.1 \mathrm{~nm}$ and $7.8 \mathrm{~nm}$, respectively. As the drift of the piezo stage is estimated to be less than $0.15 \mathrm{~nm} \mathrm{~s}^{-1}$, the effect of drift on the resolution is approximately $1 \mathrm{~nm}$. The difference between the two axes might be caused by the inhomogeneous intensity distribution of the doughnut laser beam center or the drift of the
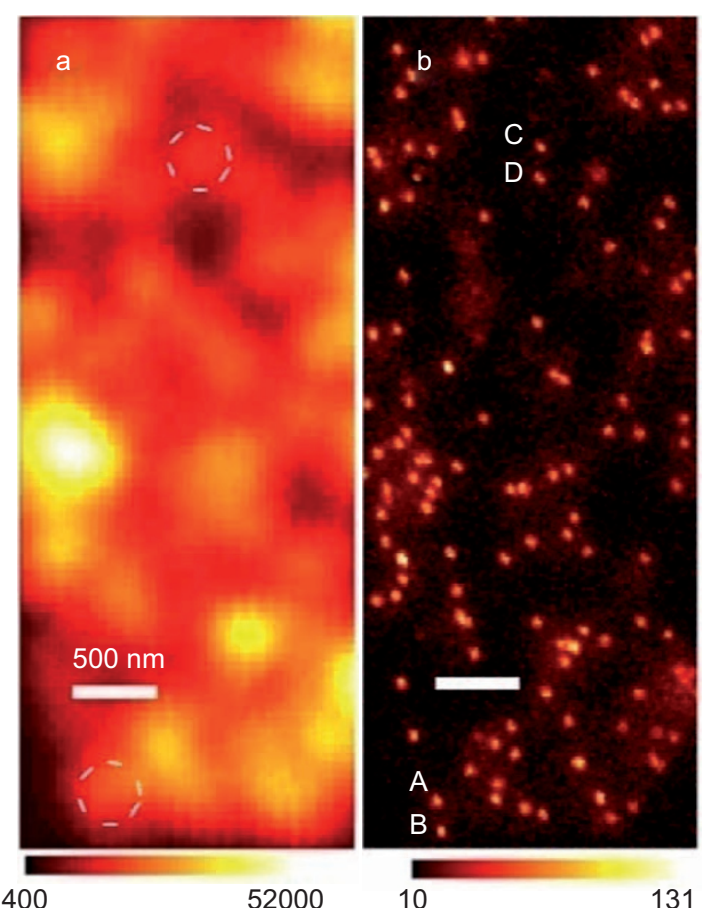

C ${ }^{3} \mathrm{E}$

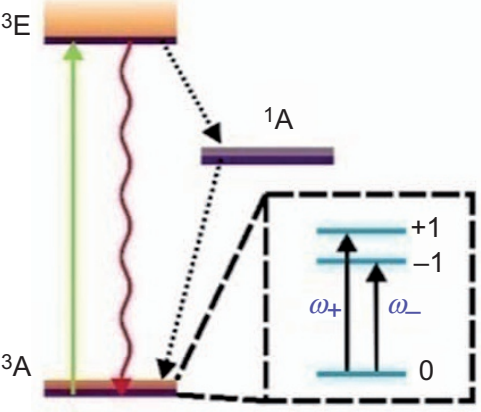

d

Confocal ODMR

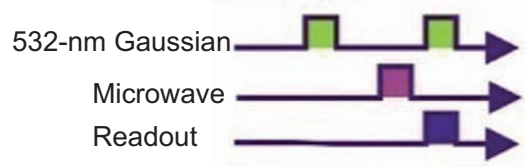

rCSD ODMR

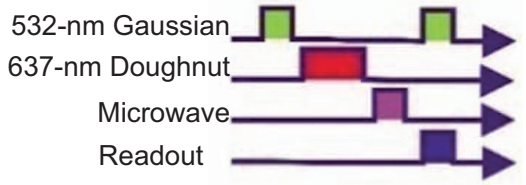

e

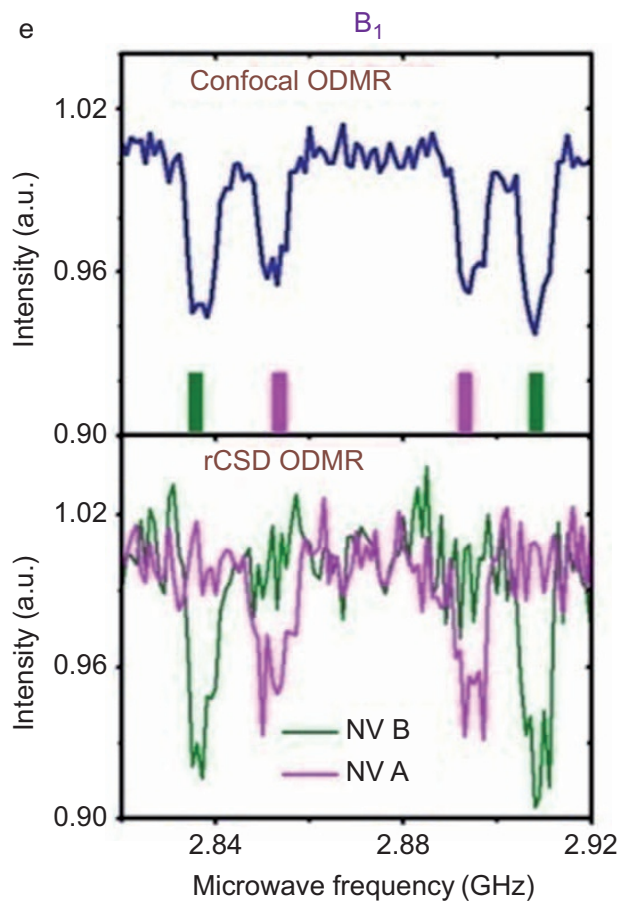

$f$

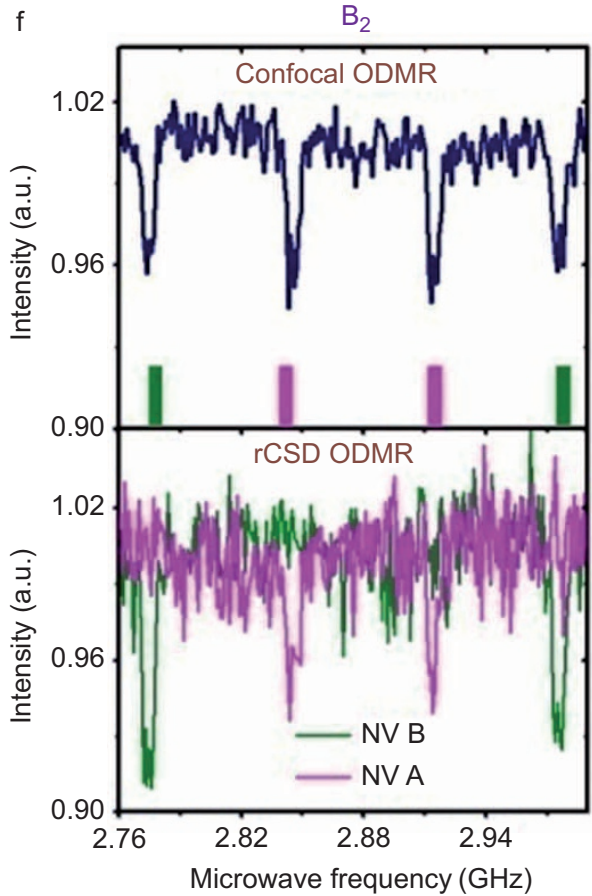

Figure 3 ( $\mathbf{a}$ and $\mathbf{b}$ ) Confocal microscopy and rCSD microscopy images of the same area in the diamond sample. The confocal microscopy images were obtained by pumping and detecting NV with a 532-nm Gaussian beam laser. The rCSD microscopy images were obtained using method shown in Figure 2b. The D laser was a 22-

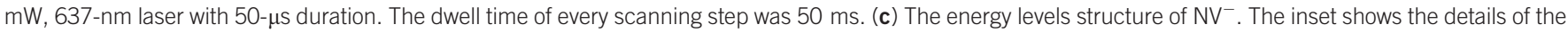
triplet ground state. (d) The pulse sequence used to detect the ODMR signals. The signal of ODMR was detected by a 0.7-mW, 532-nm laser with 300-ns duration. (e and f) The ODMR signals of two NV centers. The resonant frequencies are marked with pink (NV A) and green (NV B) bars. With the magnetic field in $\mathbf{e}$, the resonant frequencies were $\omega_{+, \mathrm{A}}=2.894 \mathrm{GHz}, \omega_{\mathrm{A}_{\mathrm{A}}}=2.852 \mathrm{GHz}, \omega_{+, \mathrm{B}}=2.908 \mathrm{GHz}, \omega_{, \mathrm{B}}=2.837 \mathrm{GHz}$. In f the resonant frequencies were $\omega_{+, \mathrm{A}}=2.914 \mathrm{GHz}, \omega_{-\mathrm{A}}=2.845 \mathrm{GHz}$, $\omega_{+, \mathrm{B}}=2.975 \mathrm{GHz}, \omega_{-, \mathrm{B}}=2.774 \mathrm{GHz}$. NV, nitrogen vacancy; ODMR, optically detected magnetic resonance; rCSD, recharging-charge-state depletion. 
stage. $^{22}$ To further improve the spatial resolution, a high numerical aperture oil objective could be used in the future. Such an objective would produce a higher charge-state conversion rate gradient than a dry objective.

Similar to the STED method, CSD can resolve the location of highdensity NV centers better than confocal microscopy (Figure $3 \mathrm{a}$ and $3 b)$. The lasers for charge-state conversion of CSD microscopy are much weaker than the lasers in STED microscopy with NV. ${ }^{19,21}$ Furthermore, our experiments demonstrate that an optimized G laser duration would also improve the spatial resolution without changing the D laser duration(see Supplementary Information for details). Therefore, the durations of both the G laser and D laser should be carefully adjusted to improve the imaging quality.

\section{Spin-state detection}

We have demonstrated that the NV center charge state can be controlled using CSD microscopy, which enables us to detect the spin state of NV with subdiffraction spatial resolution. The energy levels of the $\mathrm{NV}^{-}$triplet ground state, as shown in Figure $3 \mathrm{c}$, can be changed by the magnetic field and temperature. The two resonant frequencies of the spin-state transition can be detected using the optically detected magnetic resonance (ODMR) method. To compare the difference between CSD ODMR and confocal ODMR, we detected the ODMR signals of two adjacent NV centers with different symmetry axes, indicated as NV A and NV B in Figure 3b. The pulse sequences are shown in Figure 3d. The 532-nm Gaussian beam laser was used for both initialization and detection of the NV center. Using the 532-nm laser a

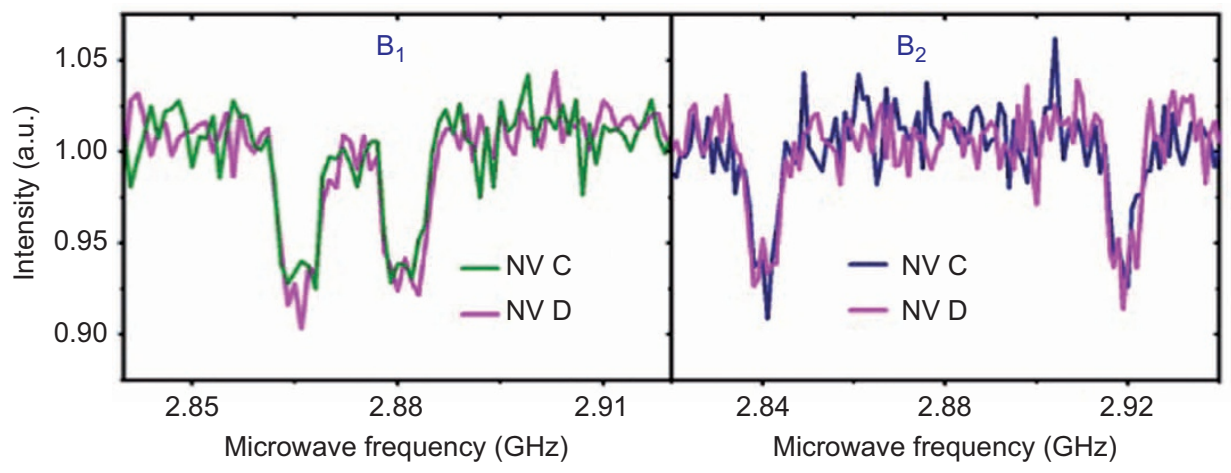

b

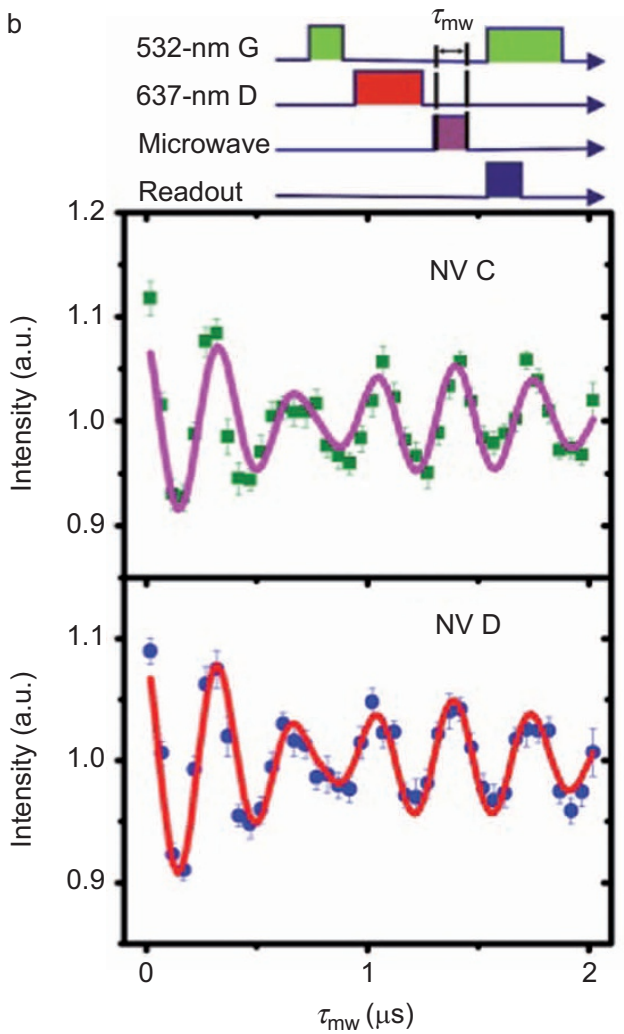

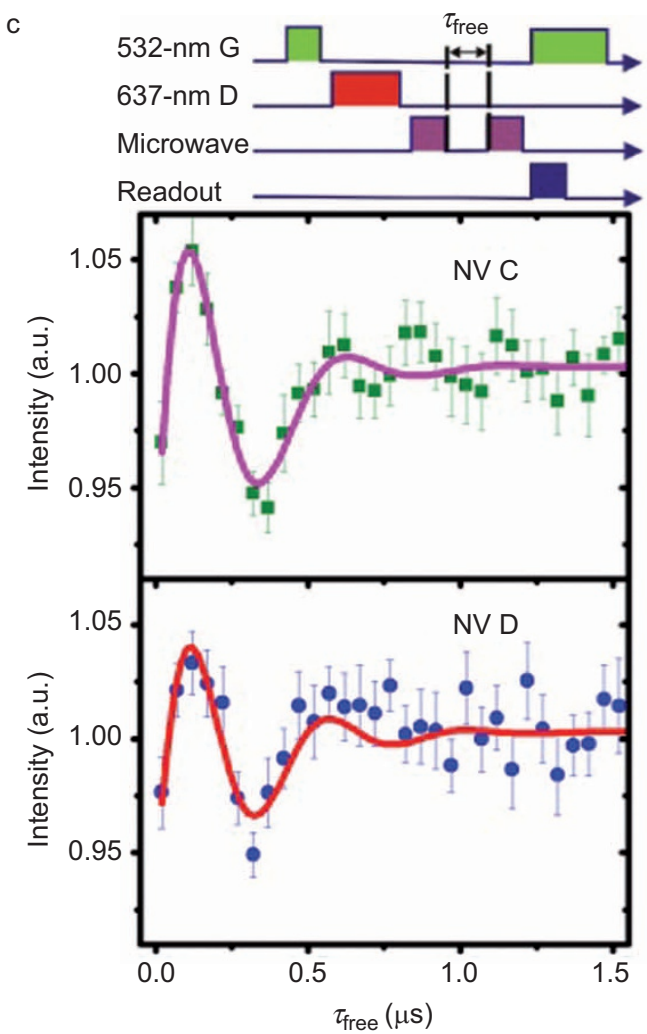

Figure 4 (a) rCSD ODMR signals of NV C and NV D in Figure 3. The different ODMR results for different magnetic fields revealed that the two NV centers had the same symmetry axis. (b) The electron spin-state Rabi oscillation using rCSD. (c) The Ramsey fringes of the NV ${ }^{-}$spin states using the rCSD method. NV, nitrogen vacancy; ODMR, optically detected magnetic resonance; rCSD, recharging-charge-state depletion. 
for detection will not affect the spatial resolution of ICSD ODMR, as the contrast of the ODMR signal between different NV centers will only be determined by the charge-state population before the microwave is applied. The resonant signals with different magnetic field were measured (Figure $3 \mathrm{e}$ and $3 \mathrm{f}$ ). The confocal ODMR in the focus area did not distinguish the electron spin resonance signals of the different NV centers. In contrast, using rCSD ODMR, the resonant frequencies of each NV were separately determined. Only the NV centers at the beam center of the $\mathrm{D}$ laser will remain to be $\mathrm{NV}^{-}$and contribute to the resonant signals of rCSD ODMR.

In Figure $3 e$ and $3 f$, the noise of $\mathrm{rCSD}$ ODMR appears to be higher than that for confocal ODMR with the same acquisition time. This effect is caused by the long duration of the D laser, which compresses the time for fluorescence detection. Even though we did not detect iCSD ODMR, we expected that this method could also separate the signals for the different NV centers. The iCSD ODMR would require one more laser beam than rCSD ODMR, as the 637-nm Gaussian beam is not suitable for use for detecting the fluorescence of $\mathrm{NV}^{-}$. Here, the magnetic field was supplied by a permanent magnet with a low magnetic field gradient. Supposing the magnetic field at NV A was the same as that at NV B, we calculated the magnetic field vector in Figure $3 \mathrm{e}^{40}$ to have magnitude $\left|\boldsymbol{B}_{1}\right| \approx 25.5 \mathrm{G}$ and direction $\theta_{1, \mathrm{~A}} \approx 73^{\circ}$, $\theta_{1, \mathrm{~B}} \approx 60^{\circ}$. Here, $\theta$ represents the angle between the magnetic field and the symmetry axis of the NV center. Another magnetic field in Figure $3 \mathrm{f}$ was $\left|\boldsymbol{B}_{2}\right| \approx 48.8 G, \theta_{2, \mathrm{~A}} \approx 76^{\circ}, \theta_{2, \mathrm{~B}} \approx 42^{\circ}$. The CSD ODMR results revealed the change in both the magnitude and direction of the magnetic field.

Although STED ODMR is also able to detect resonant frequencies of NV centers with subdiffraction resolution, ${ }^{12}$ the principles of STED ODMR and CSD ODMR are different. In the STED method, the resonant microwave pulses affect all of the NV centers; however, only one NV center's fluorescence is detected. Unlike STED ODMR, the CSD ODMR high-resolution resonant signal detection relies on charge-state manipulation. If an $\mathrm{NV}$ center is at $\mathrm{NV}^{0}$ charge state, no ODMR signal will be detected. The difference between STED ODMR and CSD ODMR might emerge in the results of coupled NV centers. For NV A and NV B in Figure 3, the resonant frequencies are different. The spin states of these two $\mathrm{NV}^{-}$centers can be separately controlled using different frequencies of microwave pulses even without the help of CSD microscopy. However, NV C and NV D in Figure $3 \mathrm{~b}$ have the same resonant frequency (Figure $4 \mathrm{a}$ ).

To selectively detect the electron spin-state dynamics of each NV center, we used the CSD method. The Rabi oscillation of an individual $\mathrm{NV}$ center was measured using the rCSD method, as shown in Figure $4 \mathrm{~b}$. The individual Ramsey fringe signals were also detected to obtain spin-state coherence information (Figure 4c). Both of the NV centers exhibited short spin coherence times, which might result from the local implantation damage. ${ }^{41}$ As the distance between these two NV centers was larger than $100 \mathrm{~nm}$, the effect of dipole interaction was not observed. The results demonstrate that the spin-state information of NV can be detected with subdiffraction spatial resolution using CSD microscopy.

The charge-state conversion will destroy the information on the NV spin state. However, recent experiments have demonstrated that the charge-state conversion of NV centers could induce a detectable change in the local field in diamond, ${ }^{2,26}$ which might affect the dynamics of the spin system that is coupled to the NV center. Subsequently, we expect that CSD could enable us to study the NV center spin-state dynamics by changing the charge state of adjacent NV centers in future work. We can use different D lasers to change the adjacent $\mathrm{NV}$ to $\mathrm{NV}^{-}$or $\mathrm{NV}^{0}$ and maintain the charge state of $\mathrm{NV}$ at the beam center to be $\mathrm{NV}^{-}$. This proposal requires us to maintain the spin state of $\mathrm{NV}$ at the beam center while changing the charge state of other NVs. However, for the lasers used in this experiment, the charge-state conversion is usually much slower than the $\mathrm{NV}^{-}$spin-state polarization. ${ }^{30}$ The spin state of $\mathrm{NV}^{-}$might be polarized to $m_{\mathrm{s}}=0$ before we can change the charge state of an adjacent NV center. To solve this problem, one method would be to combine CSD microscopy with spin-RESOLFT microscopy. ${ }^{14}$ Another method would be to use a short wavelength laser (e.g., $405 \mathrm{~nm}$ ) as the D laser. Because the NV center will not be pumped to the excited state of $\mathrm{NV}^{-}$during a singlephoton charge-state conversion process, ${ }^{25,30}$ the spin state of $\mathrm{NV}^{-}$ would not be changed before the charge state is switched.

\section{CONCLUSIONS}

In summary, we demonstrated subdiffraction charge-state manipulation of the NV centers in diamond. Laser beams with different wavelengths and shapes were used to control and detect the charge state. The best spatial resolution achieved in our experiment is approximately $4.1 \mathrm{~nm}$, which can be further improved. Using CSD microscopy, we measured the resonance signal and coherent dynamics of a NV center spin state with subdiffraction spatial resolution, which could be used for nanoscale sensing. In the future, we expect that high-resolution NV charge-state manipulation could help to control the spin-state dynamics of NVs and to switch interactions between NV centers.

\section{ACKNOWLEDGEMENTS}

This study was financially supported by the National Basic Research Program of China (Grant No. 2011CB921200), the Knowledge Innovation Project of Chinese Academy of Sciences (Grant No. 60921091), the National Natural Science Foundation of China (Grant No. 11374290), the Program for New Century Excellent Talents in University, the Fundamental Research Funds for the Central Universities and the Foundation for the Author of National Excellent Doctoral Dissertation of China.

1 Jelezko F, Gaebel T, Popa I, Gruber A, Wrachtrup J. Observation of coherent oscillations in a single electron spin. Phys Rev Lett 2004; 92: 076401.

2 Maurer PC, Kucsko G, Latta C, Jiang L, Yao NY et al. Room-temperature quantum bit memory exceeding one second. Science 2012; 336: 1283-1286.

3 Ladd TD, Jelezko F, Laflamme R, Nakamura Y, Monroe $\mathrm{C}$ et al. Quantum computers. Nature 2010; 464: 45-53.

4 van der Sar T, Wang ZH, Blok MS, Bernien H, Taminiau TH et al. Decoherenceprotected quantum gates for a hybrid solid-state spin register. Nature 2012; 484: 82-86.

5 Balasubramanian G, Chan IY, Kolesov R, Al-Hmoud M, Tisler J et al. Nanoscale imaging magnetometry with diamond spins under ambient conditions. Nature 2008; 455: 648-651.

6 Taylor JM, Cappellaro P, Childress L, Jiang L, Budker D et al. High-sensitivity diamond magnetometer with nanoscale resolution. Nat Phys 2008; 4: 810-816.

7 Kucsko G, Maurer PC, Yao NY, Kubo M, Noh HJ et al. Nanometre-scale thermometry in a living cell. Nature 2013; 500: 54-58.

8 Neumann P, Jakobi I, Dolde F, Burk C, Reuter R et al. High-precision nanoscale temperature sensing using single defects in diamond. Nano Lett 2013; 13: 2738 2742.

9 McGuinness LP, Yan Y, Stacey A, Simpson DA, Hall LT et al. Quantum measurement and orientation tracking of fluorescent nanodiamonds inside living cells. Nat Nano 2011; 6: 358-363.

10 Halla L, Simpsona D, Hollenberga L. Nanoscalesensing and imaging in biology using the nitrogen-vacancy center in diamond. MRS Bull 2013; 38: 162-167.

11 Hall LT, Beart GC, Thomas EA, Simpson DA, McGuinness LP et al. High spatial and temporal resolution wide-field imaging of neuron activity using quantum NV-diamond. Sci Rep 2012; 2: 401

12 Wildanger D, Maze JR, Hell SW. Diffraction unlimited all-optical recording of electron spin resonances. Phys Rev Lett 2011; 107: 017601.

13 Dolde F, Jakobi I, Naydenov B, Zhao N, Pezzagna S et al. Room-temperature entanglement between single defect spins in diamond. Nat Phys 2013; 9: 139-143. 
14 Maurer PC, Maze JR, Stanwix PL, Jiang L, Gorshkov AV et al. Far-field optical imaging and manipulation of individual spins with nanoscale resolution. Nat Phys 2010; 6: 912-918.

15 Han KY, Kim SK, Eggeling C, Hell SW. Metastable dark states enable ground state depletion microscopy of nitrogen vacancy centers in diamond with diffractionunlimited resolution. Nano Lett 2010; 10: 3199-3203.

16 Cui JM, Sun FW, Chen XD, Gong ZJ, Guo GC. Quantum statistical imaging of particles without restriction of the diffraction limit. Phys Rev Lett 2013; 110: 153901.

17 Chen EH, Gaathon O, Trusheim ME, Englund D. Wide-field multispectral superresolution imaging using spin-dependent fluorescence in nanodiamonds. Nano Lett 2013; 13: 2073-2077.

18 Hell SW. Far-field optical nanoscopy. Science 2007; 316: 1153-1158.

19 Han KY, Willig KI, Rittweger E, Jelezko F, Eggeling C et al. Three-dimensional stimulated emission depletion microscopy of nitrogen-vacancy centers in diamond using continuous-wave light. Nano Lett 2009; 9: 3323-3329.

20 Booth MJ. Adaptive optical microscopy: the ongoing quest for a perfect image. Light Sci App/ 2014; 3: e165; doi:10.1038/Isa.2014.46.

21 Arroyo-Camejo S, Adam MP, Besbes M, Hugonin JP, Jacques V et al. Stimulated emission depletion microscopy resolves individual nitrogen vacancy centers in diamond nanocrystals. ACS Nano 2013; 7: 10912-10919.

22 Wildanger D, Patton BR, Schill H, Marseglia L, Hadden JP et al. Solid immersion facilitates fluorescence microscopy with nanometer resolution and sub-Ångström emitter localization. Advanced Materials 2012; 24: OP309-OP313.

23 Waldherr G, Beck J, Steiner M, Neumann P, Gali A et al. Dark states of single nitrogenvacancy centers in diamond unraveled by single shot NMR. Phys Rev Lett 2011; 106: 157601.

24 Siyushev P, Pinto H, Vörös M, Gali A, Jelezko F et al. Optically controlled switching of the charge state of a single nitrogen-vacancy center in diamond at cryogenic temperatures. Phys Rev Lett 2013; 110: 167402.

25 Aslam N, Waldherr G, Neumann P, Jelezko F, Wrachtrup J. Photo-induced ionization dynamics of the nitrogen vacancy defect in diamond investigated by single-shot charge state detection. New J Phys 2013; 15: 013064.

26 Dolde F, Doherty MW, Michl J, Jakobi I, Naydenov B et al. Nanoscale detection of a single fundamental charge in ambient conditions using the $\mathrm{NV}^{-}$center in diamond. Phys Rev Lett 2014; 112: 097603.

27 Chu Y, de Leon N, Shields B, Hausmann BJ, Evans R et al. Coherent optical transitions in implanted nitrogen vacancy centers. Nano Lett 2014; 14: 1982-1986.

28 Waldherr G, Neumann P. Huelga SF, Jelezko F, Wrachtrup J. Violation of a temporal bell inequality for single spins in a diamond defect center. Phys Rev Lett 2011; 107: 090401.
29 Gaebel T, Domhan M, Wittmann C, Popa I, Jelezko F et al. Photochromism in single nitrogen-vacancy defect in diamond. Appl Phys B 2006; 82: 243-246.

30 Chen XD, Zou CL, Sun FW, Guo GC. Optical manipulation of the charge state of nitrogen-vacancy center in diamond. Appl Phys Lett 2013; 103: 013112.

31 Neumann P, Kolesov R, Naydenov B, Beck J, Rempp F et al. Quantum register based on coupled electron spins in a room-temperature solid. Nat Phys 2010; 6: 249-253.

32 Doi Y, Makino T, Kato H, Takeuchi D, Ogura M et al. Deterministic electrical chargestate initialization of single nitrogen-vacancy center in diamond. Phys Rev X2014; 4: 011057.

33 Balasubramanian G, Neumann P, Twitchen D, Markham M, Kolesov R et al. Ultralong spin coherence time in isotopically engineered diamond. Nat Mater 2009; 8: 383387.

34 Kato H, Wolfer M, Schreyvogel C, Kunzer M, Müller-Sebert W et al. Tunable light emission from nitrogen-vacancy centers in single crystal diamond PIN diodes. App/ Phys Lett 2013; 102: 151101.

35 Hauf MV, Grotz B, Naydenov B, Dankerl M, Pezzagna S et al. Chemical control of the charge state of nitrogen-vacancy centers in diamond. Phys Rev B 2011; 83: 081304(R).

36 Beha K, Batalov A, Manson NB, Bratschitsch R, Leitenstorfer A. Optimum photoluminescence excitation and recharging cycle of single nitrogen-vacancy centers in ultrapure diamond. Phys Rev Lett 2012; 109: 097404.

37 Hauf MV, Simon P, Aslam N, Pfender M, Neumann P et al. Addressing single nitrogenvacancy centers in diamond with transparent in-plane gate structures. Nano Lett 2014; 14: 2359-2364.

38 Westphal V, Hell SW. Nanoscale resolution in the focal plane of an optical microscope. Phys Rev Lett 2005; 94: 143903.

39 Hell SW. Strategy for far-field optical imaging and writing without diffraction limit. Phys Lett A 2004; 326: 140-145.

40 Chen XD, Sun FW, Zou CL, Cui JM, Zhou LM et al. Vector magnetic field sensing by a single nitrogen vacancy center in diamond. Europhys Lett 2013; 101: 67003.

41 Yamamoto T, Müller C, McGuinness LP, Teraji T, Naydenov B et al. Strongly coupled diamond spin qubits by molecular nitrogen implantation. Phys Rev B 2013; 88: 201201

(c) (i) (2) This work is licensed under a Creative Commons AttributionNonCommercial-ShareAlike 3.0 Unported License. The images or other third party material in this article are included in the article's Creative Commons license, unless indicated otherwise in the credit line; if the material is not included under the Creative Commons license, users will need to obtain permission from the license holder to reproduce the material. To view a copy of this license, visit http://creativecommons.org/licenses/by-nc-sa/3.0/

Supplementary information for this article can be found on the Light: Science \& Applications' website (http://www.nature.com/lsa/). 